\title{
THE
}

\section{Exercise and Self-Esteem: Validity of Model Expansion and Exercise Associations}

\author{
Robert J. Sonstroem \\ University of Rhode Island \\ Lisa L. Harlow \\ University of Rhode Island, Iharlow@uri.edu \\ Lynn Josephs
}

Follow this and additional works at: https://digitalcommons.uri.edu/psy_facpubs

Terms of Use

All rights reserved under copyright.

\section{Citation/Publisher Attribution}

Sonstroem, R. J., Harlow, L. L. \& Josephs, L. 1994. Exercise and Self Esteem: Validity of Model Expansion and Exercise Associations. Journal of Sport \& Exercise Psychology, 16(1), 29-42. doi: 10.1123/

jsep.16.1.29

Available at: http://dx.doi.org/10.1123/jsep.16.1.29

This Article is brought to you for free and open access by the Psychology at DigitalCommons@URI. It has been accepted for inclusion in Psychology Faculty Publications by an authorized administrator of DigitalCommons@URI. For more information, please contact digitalcommons-group@uri.edu. 


\title{
Exercise and Self-Esteem: Validity of Model Expansion and Exercise Associations
}

\author{
Robert J. Sonstroem, Lisa L. Harlow, and Lynn Josephs \\ University of Rhode Island
}

\begin{abstract}
The purpose of this research was to test expansion of the Exercise and Self-Esteem Model (EXSEM) to include two levels of perceived physical competence as operationalized by the Physical Self-Perception Profile (PSPP). Female aerobic dancers $(N=216$, age $M=38.4)$ were administered a Self-Esteem scale (SE), the PSPP to assess a general Physical Self-Worth (PSW), and more specific subdomains of perceived Sport Competence (Sport), Physical Condition (Cond), Attractive Body (Body), and Strength (Stren). Subjects also completed self-efficacy scales for jogging, sitting, and aerobic dancing. Confirmatory factor analysis supported model measurement as hypothesized, $\chi^{2}=1,154.88, \mathrm{df}=681$, comparative fit index $(\mathrm{CFI})=.913$, root mean square residual $(\mathrm{RMSR})=.047$. Structural equation modeling (SEM) supported EXSEM component relationships as proposed. Further SEM associating two exercise self-reports with EXSEM again displayed satisfactory fit indices and explained up to $27.6 \%$ of exercise variance. It was concluded that exercise in adult female aerobic dancers is associated with positive evaluations of their physical condition and with negative evaluations of their bodies.
\end{abstract}

Key words: perceived physical competence, self-esteem model, exercise prediction

Sonstroem and Morgan (1989) developed an Exercise and Self-Esteem Model (EXSEM) based on contemporary theory, proposing that self-concept is best studied as a collection of self-perceptions organized on hierarchical levels of specificity/generality (e.g., Shavelson, Hubner, \& Stanton, 1976). Within its vertical competence continuum, the EXSEM proposes that self-efficacies of one's abilities to perform specific exercise or sport-training activities generalize to a broader perceived physical competence. Global self-esteem represents the highest, most general, level of the model. The model was developed to trace the manner

Robert J. Sonstroem is with the Department of Physical Education, and Lisa L. Harlow is with the Department of Psychology at the University of Rhode Island, Kingston, RI 02881. Lynn Josephs is with the Heathsouth Rehabilitation Center, 2919 Arlington Dr., Alexandria, VA 22306. 
in which self-esteem is influenced by physical training activities. A model of this nature is believed to offer greater heurism and is in agreement with current theory that examines self-perception profiles and hierarchies rather than a unidimensional self-concept (Harter, 1985; Marsh, Byrne, \& Shavelson, 1988).

Structural modeling analyses have validated the model structure as proposed, and the model was able to explain $29 \%$ of global self-esteem variance (Sonstroem, Harlow, Gemma, \& Osborne, 1991). More recent research tested an adaptation of the model with data collected over three time periods across a varsity high school swim season (Sonstroem, Harlow, \& Salisbury, 1993). Structural modeling analysis over these three time waves revealed an excellent fit of model to data, $\chi^{2}=25.46, \mathrm{df}=19, p>.05$, root mean square residual (RMSR) $=.039$. Also of interest was the observed capability of perceived physical competence at Time 1 to significantly predict swimming performance at Time 2 . This association supports the self-enhancement theory of self-esteem (i.e., we tend to act in ways that support our perceptions of ourselves). Previously, Marsh (1990) has demonstrated that academic self-concept scores can significantly influence subsequent academic achievement scores.

Of additional importance in the Sonstroem et al. (1993) study is the fact that no model relationships during the swim season were significantly influenced by socially desirable responding assessed at the three time periods. Self-esteem, as well as perceived physical competence, exerted predictive priority over the influence of social desirability. Previous validation efforts, as well as the present research, have been delimited to testing the competence dimension of the model. The two validation studies cited above employed study-developed modifications of the Estimation Scale (Sonstroem, 1978) to assess a unidimensional perceived physical competence.

Meanwhile, Fox and Corbin (1989) have developed the Physical SelfPerception Profile (PSPP), which separates perceived physical competence into general physical self-worth (PSW), subsuming more domain-specific scales of Sport Competence (Sport), Physical Condition (Cond), Attractive Body (Body), and Strength (Stren). Instrument development with college students demonstrated excellent internal consistencies for the five scales. These also manifested hypothesized associations with self-reported type and intensity of exercise. Moreover, PSW was significantly related to the four subdomain scales and, as hypothesized, was more highly related to global self-esteem than were the subdomain scales. The PSPP scales offer advantages to the exercise scientist in that they greatly expand the description of perceived physical competence and provide immediate opportunities for developing discriminant, as well as convergent, validity.

Sonstroem, Speliotis, and Fava (1992) validated the PSPP for use with males and females in their middle years (age $M=44.1$ ). Exploratory factor analysis of the four subdomain scales revealed near perfect separation of items into hypothesized components and very high factor loadings. Scales, and especially Cond, were able to predict self-reports of degree of physical activity very well (Canon. $R=.73$ for females, Canon. $R=.64$ for males).

The purpose of the present research was to test the validity of expanding the EXSEM to include two levels of perceived physical competence as operationalized by the PSPP. This involved assessing the fit of data to one level of selfefficacies, one level of PSPP subdomains, a third level of PSW, and a final level of global self-esteem. Confirmatory factor analyses (CFA) and structural equation 
modeling with item level data provided a test of construct measurement, as well as tests of proposed pathways within the model. Earlier validation of the model (Sonstroem et al., 1991) summed item responses into scales before structural equation modeling and tested the latent variables of perceived physical competence and self-esteem by use of two measures (scales). An item-level analysis, as employed in the present research, is regarded as more sensitive to departures in assessment or prediction.

A second purpose of this study was to analyze the measurement properties of the PSPP by means of CFA. Though Fox and Corbin (1989) report use of CFA, PSW was not included in their analysis. The Sonstroem et al. (1992) study identified a large amount of overlap between the PSW and Body scales in the exploratory factor analysis and in scale intercorrelations (i.e., Pearson $r=+.759$ ). This indicates a preponderance of shared variance in each construct (i.e., $r^{2}>.50$ ).

A third purpose of this research was to bring further study to latent variable relationships within model structure. Essential to a self-system such as the EXSEM is a delineation of pathways between elements within the model, as well as the determination of element responsibilities for relationships with variables outside the model. In terms of associations within the model, several considerations arose. First, because of Body-PSW overlap, it was important to examine the necessity of including PSW within the model. A parallelism in their associations with self-esteem suggests that a good data fit and a more parsimonious model might be obtained by PSW deletion. A second consideration involved determining relationships between constructs positioned in nonadjacent levels of specificity. The Sonstroem et al. (1993) study demonstrated a better data fit when model associations were permitted up to two vertical levels distant from each other. However, the Sonstroem et al. (1991) study failed to develop significant associations between self-efficacies and self-esteem. Structural modeling analyses were employed in the present research to address the two problems above.

Associations with variables outside the model are relevant to this last argument. The Sonstroem-Morgan model, based on theory of Shavelson et al. (1976), specifies that lower level, more specific self-evaluations (i.e., self-efficacies) will be most closely related to actual behavior in the environment. However, Sonstroem et al. (1993) found that perceived physical competence, rather than more specific self-perceptions, predicted subsequent swim performance. The EXSEM has been developed from a skill-development perspective that denotes an upward flow of competence generalizing within the model from more specific to more general self-evaluations. However, in terms of predicting external behavior, and while utilizing self-efficacy in this prediction, the model may also incorporate pervasive self-enhancement capacities of more general self-evaluations. The present study provided an indirect test of self-enhancement theory by examining the ability of model latent variables to develop associations with selfreports of exercise behavior obtained concurrently.

\section{Procedure}

Subjects consisted of 216 adult female aerobic dancers contacted at aerobic dance classes in Washington, DC, and Providence, RI (Age $M=38.4, S D=$ 16.2). Subjects signed an informed consent and completed the questionnaire anonymously. 


\section{Instruments}

Self-Esteem (SE). Self-esteem was assessed with the 6-item General SelfWorth scale of the Adult Self-Perception Profile (Messer \& Harter, 1986). This and PSPP scales employ a 4-point structured alternative format designed to reduce socially desirable responses. Subjects indicate which of two contrasting descriptions is most like them and the degree of similarity.

Physical Self-Perception Profile (PSPP). The PSPP contains 30 items subdivided into 6-item scales assessing self-perceptions of Physical Self-Worth (PSW), Sport Competence (Sport), Physical Condition (Cond), Attractive Body (Body), and Strength (Stren) (Fox, 1990; Fox \& Corbin, 1989). Its reliability and validity as used for middle-aged adults has been supported (Sonstroem et al., 1992). Alpha coefficients in the latter study were all either .90 or .91 for females.

Self-Efficacy. Three single-item scales were written for self-efficacies regarding jogging, sit-ups, and aerobic dancing following procedures advocated by Bandura and Adams (1977). Subjects indicated how confident they were (scale of 0 to 100) at jogging for distances (11 different distances up to 8 miles were presented); at performing repetitive sit-ups ( 8 categories up to 120 repetitions); at vigorous aerobic dancing ( 9 different time durations up to 180 minutes). Strength of self-efficacy scores were calculated for each event by summing degrees of confidence for that self-efficacy and dividing by the number of categories on that particular scale. For SEM purposes the present study hypothesizes that these three measures contribute to a latent variable, efficacy (Eff).

Physical Activity Participation. Subjects were asked to indicate "the number of aerobic classes they had attended in the past 10 weeks.' Responses were $<11,11-20,20-25,26-30$, and $>30$. Excepting aerobic dance classes, they also were asked how many times per week they engaged "in vigorous physical activity long enough to work up a sweat." Responses were $\langle 1,1,2,3$, and $>$ 3. Subjects also indicated the average duration of time exercised in activities other than aerobic dance. Responses were <11 minutes, 11 to 20 minutes, 21 to 30 minutes, 31 to 45 minutes, and $>45$ minutes.

Structural Modeling Analyses. The EQS computer program (Bentler, 1989) was employed with a maximum likelihood solution. This procedure has been shown to be robust to departures from normality (Harlow, 1985; Huba \& Harlow, 1987). Because no index exists that is best at identifying overall model fit (Bentler, 1990), the following recommended statistics were all employed. Indicative of a good model-to-data fit are (a) a chi-square that is small in comparison to its degrees of freedom; (b) the comparative fit index (CFI) (Bentler, 1990), which should have values approaching unity; (c) the parsimonious CFI, which controls for model complexity (James, Mulaik, \& Brett, 1982); and (d) the root mean square residual (RMSR), which should approach zero.

Pathways within the model were tested for significance $(p<.05)$ and provided with standardized parameter estimates. These include paths between hypothesized latent constructs and item measures (i.e., factor loadings) and hypothesized paths among latent constructs (i.e., regression coefficients). Additionally, it is possible to determine model validity by examining the percentage of variance accounted for in each of the dependent variables (i.e., 1 - prediction error). 


\section{Structural Models}

Measurement Model. The 39 self-perception variables were proposed as measures of the seven latent variables spanning four levels of (a) SE; (b) PSW; (c) Sport, Cond, Body, and Stren; and (d) Eff. Within this and subsequent models tested, a single variable was arbitrarily selected as a reference point for each hypothesized factor, and its factor loading was fixed at 1.0. Variances of latent variables were freely estimated, as were covariances among factors. Altemative measurement models were tested also, including one that examined the effect of considering Body and PSW items as measures of a single factor.

Model $A$. This presents the competence dimension of the EXSEM, as originally conceived, with the addition of a second level of specific subdomains of perceived physical competence (see Figure 1). Pathways are proposed as developing only between latent variables adjacent on the vertical continuum (e.g., Cond and PSW, but not Cond and SE). Covariances among disturbances at the subdomain level were freely estimated.

Within the proposed EXSEM (see Figure 1, Model 1), it would be expected that Eff representing self-efficacies of vigorous exercise (i.e., jogging, situps, dancing) would explain amounts of variance in Sport, Cond (particularly), Stren, and a lesser amount of variance in predicting Body. Although the four subdomain scales should be capable of predicting PSW with a great deal of accuracy, they should be less effective than PSW at predicting SE.

Model $B$. This model permits tests of associations among variables two levels removed from each other (Figure 1). It hypothesized paths between the four subdomains and SE and between Eff and PSW in addition to all of the paths hypothesized in Model A.

Models $C$ and $D$. Because of the previously reported overlap between Body and PSW scales, Models C and D were analyzed to test the necessity of including PSW as a mediator between subdomains and SE. Model C differs from Model D only in that it includes a path from PSW to SE. It was agreed a priori that if Model D were to display a data fit equal to Model $\mathrm{C}$ and could explain as much SE variance as Model C, the inclusion of PSW in the model could be discounted.

\section{Physical Activity Participation}

Originally, it was planned to construct a single activity composite for the three measures-aerobic class attendance, frequency of other activity, and typical duration of other activity. However, both frequency and duration of other activity proved to be inversely related to class attendance, thus limiting the convergence of covariance solutions employing these three as measures of a single latent variable. Therefore, two methods of estimating physical activity were used. In one, the measure of class attendance was employed as a single indicator of the latent variable dance. Its error was fixed at zero, and its disturbance was freely estimated. The second method employed frequency and duration of other activity as measures of a latent variable, exercise.

Activity Model Tested. Pertinent theory suggests that exercise should be most closely allied with the level of self-perception most specific to and congruent with itself. Therefore, dance and exercise were positioned at the Eff level in the 


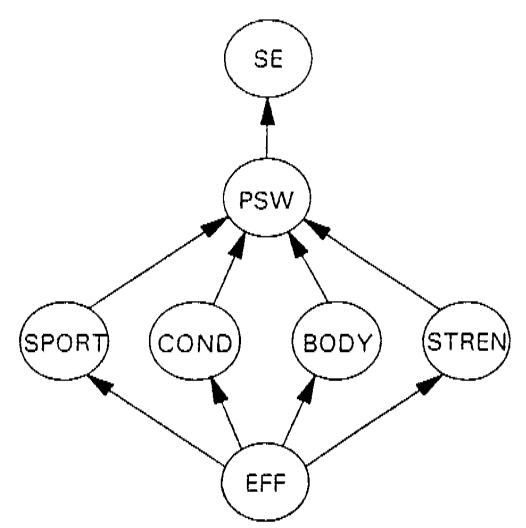

MODEL A

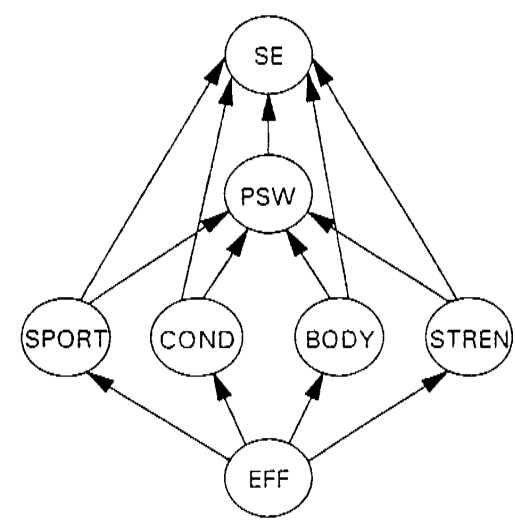

MODEL C

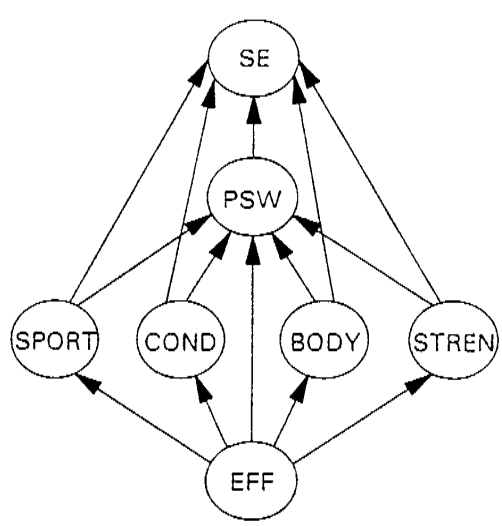

MODEL B

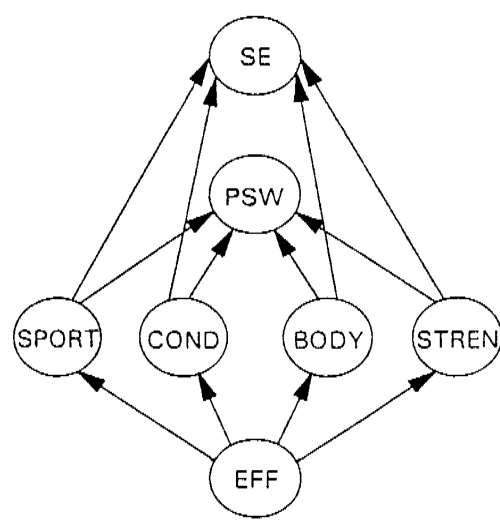

MODEL D

Figure 1 - Structural models tested.

model (Figure 2). In recognition of the self-enhancement properties of perceived physical competence, paths from subdomains to physical activity were hypothesized. In other respects the model is similar to Model A with the direction of influence reversed.

\section{Results}

The first two rows of Table 1 contain item means and standard deviations for each of this study's seven proposed latent variables. Row 3 presents Cronbach 


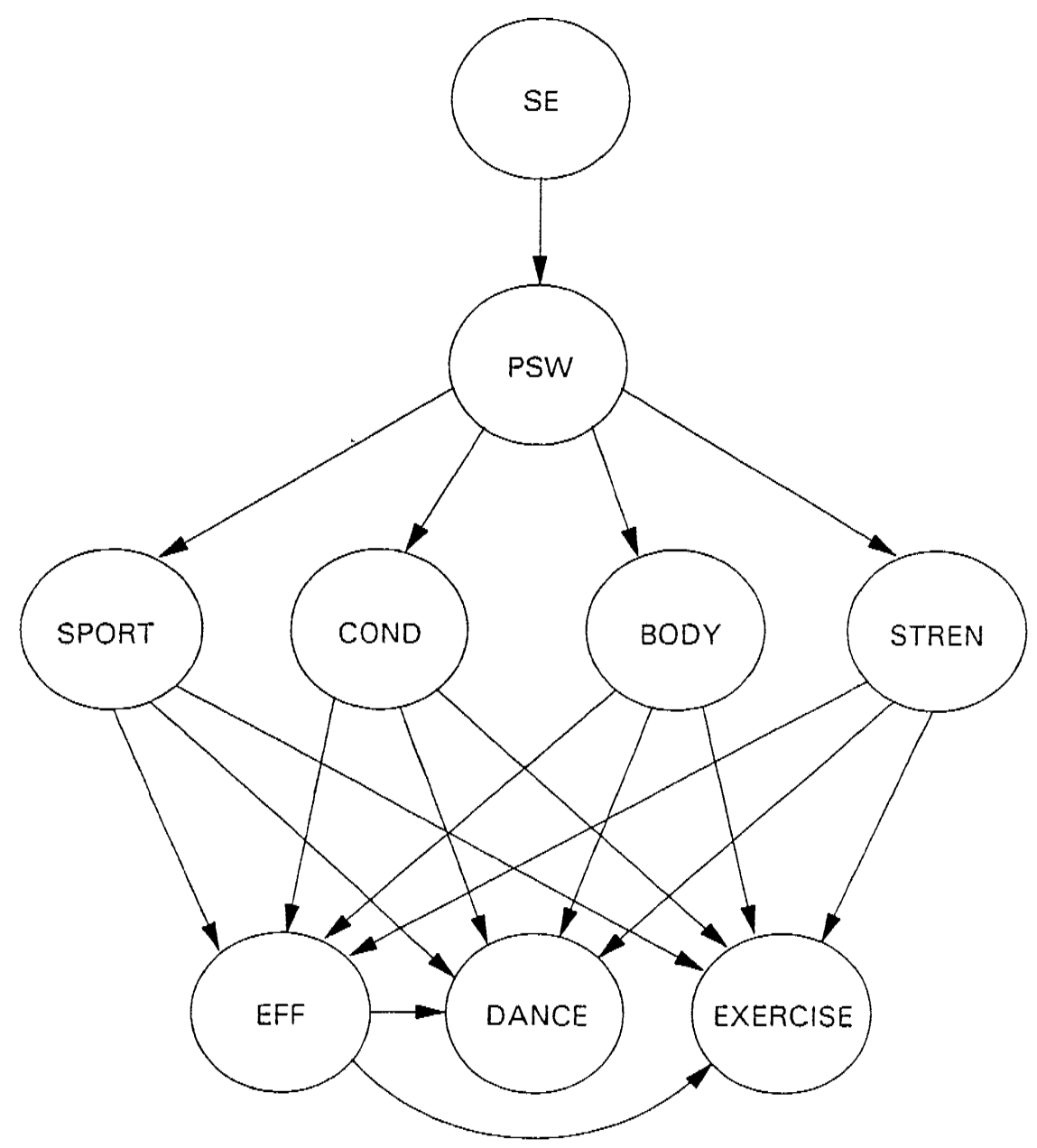

Figure 2 - Physical activity prediction model.

alpha values, which range from acceptable to excellent. CFA of the 39 selfperception items confirmed the measurement model as proposed (Table 2). A comparative fit index (CFI) of .913 , a parsimonious CFI (PCFI) of .839 and a root mean square residual of .047 are indicative of a satisfactory fit of data to model. The lower portions of Table 1 contain a matrix of latent variable intercorrelations corrected for measurement error. It is apparent from the SE column that the essential hierarchy of EXSEM is supported in that the association between SE and PSW is larger than the average subdomain association with SE. The lowest hierarchical level (Eff) develops the smallest SE relationship. A similar pattern is manifested in the PSW column. However, the very large associations of PSW with Body $(r=.85)$ and with Cond $(r=.80)$ are disturbing. This lack of independence in the PSW and Body constructs has been commented on earlier by Sonstroem et al. (1992). 
Table 1 Factor Item Means, Standard Deviations, Cronnbach Alphas and Intercorrelations From Measurement Model

\begin{tabular}{lrrrrrrr}
\hline & SE & PSW & Sport & Cond & Body & Stren & Eff \\
\hline Item $M$ & 3.14 & 2.69 & 2.31 & 3.13 & 2.50 & 2.79 & 69.92 \\
Item $S D$ & .61 & .71 & .78 & .65 & .78 & .66 & 21.67 \\
Alpha & .70 & .87 & .92 & .88 & .91 & .90 & .84 \\
PSW & .59 & - & - & - & - & - & \\
Sport & .35 & .66 & - & - & - & - & - \\
Cond & .39 & .80 & .57 & - & - & - & \\
Body & .51 & .85 & .40 & .66 & - & - & \\
Stren & .29 & .63 & .65 & .61 & .36 & - & \\
Eff & .13 & .32 & .39 & .53 & .22 & .41 & \\
& & & & & & & \\
\hline
\end{tabular}

Note. Item $M=$ item means on a scale of $1-4$ for six items in each scale, three items in Eff scale; item $S D=$ standard deviation for the six items of each scale, three items of Eff; PSW = Physical self-worth; Sport = sport; Cond $=$ physical condition; Body $=$ attractive body; Stren = strength; Eff = self-efficacy.

Table 2 Measurement and Structural Model Fit Indices and Comparisons With Model A

\begin{tabular}{|c|c|c|c|c|c|c|c|c|}
\hline Model & $\chi^{2}$ & df & CFI & PCFI & RMSR & $\begin{array}{c}\text { No. } \\
\text { paths } \\
\text { hypoth. }\end{array}$ & $\begin{array}{l}\text { No. } \\
\text { paths } \\
\text { sig. }\end{array}$ & $\begin{array}{c}\% \mathrm{SE} \\
\text { explained }\end{array}$ \\
\hline Meas. & $1,154.88$ & 681 & .913 & .839 & .047 & - & - & - \\
\hline A & $1,162.66$ & 687 & .913 & .840 & .048 & 9 & 9 & 32.8 \\
\hline B & $1,154.97$ & 682 & .913 & .840 & .047 & 14 & 9 & 36.6 \\
\hline C & $1,158.34$ & 683 & .913 & .841 & .047 & 13 & 9 & 37.0 \\
\hline D & $1,165.97$ & 684 & .913 & .841 & .047 & 12 & 9 & 30.6 \\
\hline
\end{tabular}

Model comparisons $\quad \chi^{2}$ diff. df diff. $\quad p$

$\begin{array}{lrrr}\text { Models A-B } & 7.69 & 5 & \text { n.s. } \\ \text { Models B-C } & 4.32 & 4 & \text { n.s. } \\ \text { Models A-D } & -3.31 & 3 & \text { n.s. } \\ \text { Models C-D } & -7.63 & 1 & .01\end{array}$

Note. CFI = Comparative fit index; $\mathrm{PCFI}=$ parsimonious comparative fit index;

RMSR $=$ root mean square residual; No. paths hypoth. $=$ number of paths in the model hypothesized to be significant; No. paths sig. = number of paths which tested as significant; \% SE explained = percent of SE variance explained by the model. 


\section{Structural Equations}

Table 2 presents fit indices for the four structural models tested. The preferred model, Model A, displayed a CFI of .913, a PCFI of .840, and a RMSR of .048. Most importantly, all of its hypothesized paths were significant. Although the other three models demonstrated data fits similar to those of Model A, none proved superior to Model A when tested for significant differences (see bottom five rows of Table 2). Although Models $B$ and $C$ explained somewhat more of the variance in SE, they both manifested hypothesized paths that were not significant. The path from Eff to PSW was not significant, in Model B. As stated earlier, Models C and D were tested to examine the necessity of including PSW within the model as a mediator between subdomains and SE. As shown in the bottom row of Table 2, Model C (with PSW) demonstrated a significantly superior fit $(p<.01)$ to that of Model D (without PSW).

It was concluded that model structure and associations among model elements are best depicted by Model A because of the following considerations: (a) All hypothesized paths in Model A were significant whereas not all those in other models were; (b) other models failed to demonstrate significantly better correspondence with the data as compared Model A; and (c) the comparison of Models C and D demonstrated the necessity of including PSW within the model.

Figure 3 presents the standardized solution for Model A. Values printed beside paths represent regression coefficients standardized to a range of -1 to +1 . It can be seen that Body develops the strongest association with PSW (more than twice as great as any of the other subdomains). Values within parentheses represent the amount of variance in each construct that is explained by the model. For example, the model accounted for $32.8 \%$ of $\mathrm{SE}$ variance, which is surprising when one considers the many personal factors on which self-esteem is based.

\section{Association With Exercise Participation}

The first two rows of Table 3 present fit indices for the expanded EXSEM's association with physical activity. A CFI of .911, a PCFI of .840, and an RMSR of .047 indicate a data fit similar to that of Model A, even with the addition of three measures and two latent variables, dance and exercise. The right side of Table 3 contains standardized regression coefficients for all variables predicting exercise (Row 2). Significant predictors are starred. The structural equation predicted $26.6 \%$ of dance variance and $27.6 \%$ of exercise variance (last column to right). The right side of Table 3 indicates that of five predictors, only the same two (Cond and Body) were significantly related to each dependent variable. Cond developed standardized regression coefficients of +.69 and +.51 with dance and exercise, respectively. Body's coefficients were -.29 and -.30 with these same dependent variables. The negative signs on these coefficients indicate the probable presence of suppression effects.

According to Tabachnich and Fidell (1983), suppression occurs when an independent variable, relatively uncorrelated with the dependent variable, increases the multiple prediction of the dependent variable by its relationship with other independent variables. This serves to suppress that variance in these other independent variables, which is unrelated to the dependent variable. Symptoms of a suppressor effect may include independent variable regression weights that 


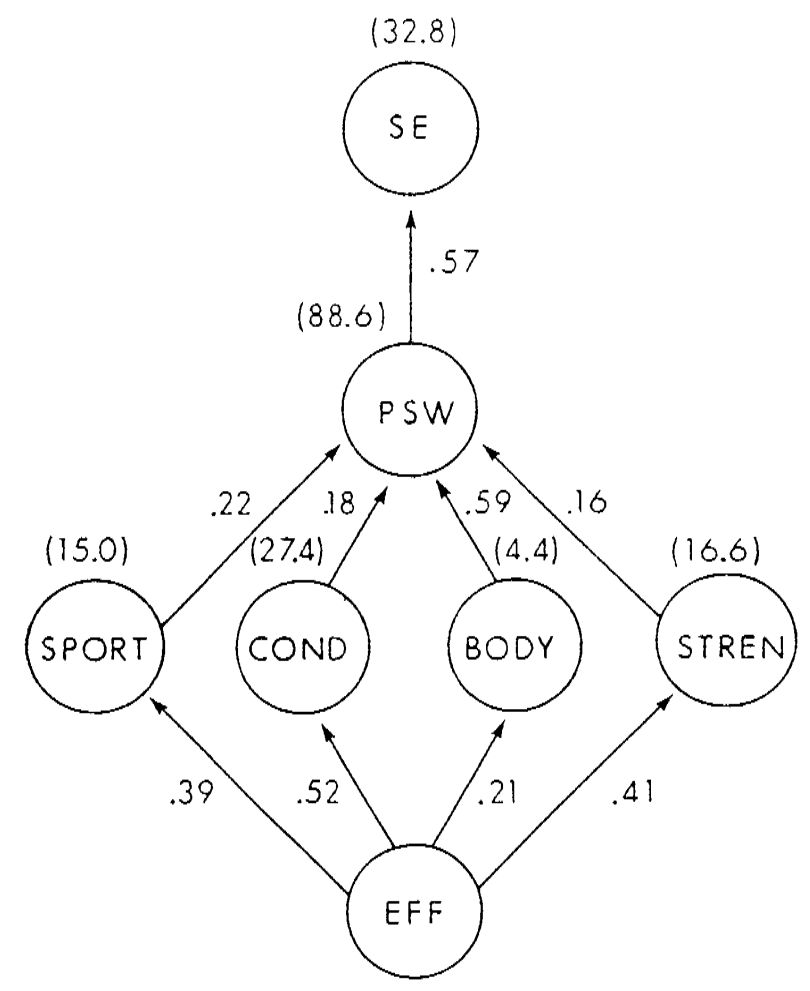

Figure 3-Standardized solution, test of Model A. Values beside paths represent standardized regression coefficients. Values within parentheses represent variance percentages accounted for.

are substantially larger than the zero-order coefficients between the independent and dependent variables, or situations in which the zero-order $r$ and the standardized regression coefficient have opposite signs. In the present case, zero-order $r$ s between dance and Sport, Cond, Body, and Stren were .05, .38, .07, and .10, respectively. Correlations between exercise, and Sport, Cond, Body, and Stren were $.37, .45, .12$, and .37 , respectively. When standardized regression coefficients of Table 3 are compared to the above zero-order coefficients, the presence of reversed signs and several inflated values (see Cond and Body) is evident.

Although suppression effects in predicting activity are noticeable, substantive content associations with activity are believed to be present as well. This may be more apparent for Cond, but is believed to be true also of Body. Cohen and Cohen (1975, p. 90) state that negative partial coefficients invariably become attached to the predictor with the initially smaller zero-order relationship. An examination of Table 1 subdomain means reveals that Body has the lowest value for all PSPP scales, with the exception of Sport. This latter subdomain would seem of small relevance to 38 -year-old women involved in aerobics classes. The importance of the body to females in the USA is recognized, however (Cash \& Green, 1986). 
Table 3 Fit Indices and Models in Associations With Physical Activity

\begin{tabular}{|c|c|c|c|c|c|c|c|c|c|c|c|}
\hline \multirow[b]{2}{*}{ Models } & \multirow[b]{2}{*}{$\chi^{2}$} & \multirow[b]{2}{*}{$\mathrm{df}$} & \multirow[b]{2}{*}{$\mathrm{CFI}$} & \multirow[b]{2}{*}{ RMSR } & \multirow[b]{2}{*}{ DV } & \multicolumn{5}{|c|}{ Predictors } & \multirow{2}{*}{$\begin{array}{c}\% \\
\text { varianc }\end{array}$} \\
\hline & & & & & & Eff & Sport & Cond & Body & Stren & \\
\hline \multirow[t]{2}{*}{ EXSEM } & $1,296.20$ & 794 & .911 & .047 & Dance & .14 & -.17 & $.69^{*}$ & $-.29 *$ & -.16 & 26.0 \\
\hline & & & $(.840)$ & & Exercise & .03 & .15 & $.51^{*}$ & $-.30 *$ & .05 & 27.6 \\
\hline \multirow[t]{2}{*}{ Refined } & 656.04 & 393 & .927 & .047 & Dance & .11 & - & $.50 *$ & $-.29 *$ & - & 19.7 \\
\hline & & & $(.837)$ & & Exercise & .05 & - & $.65^{*}$ & $-.32 *$ & - & 27.2 \\
\hline
\end{tabular}

Note . $\mathrm{CFI}=$ comparative fit index; $\mathrm{PCFI}=$ parsimonious comparative fit index;

RMSR $=$ root mean square residual; DV $=$ dependent variables. Values under predictors represent standardized regression coefficients. \% variance $=$ percentage of dependent variable variance explained by the model. Parsimonious CFI (PCFI) values are presented in parentheses below CFI values.

$*_{p}<.05$.

The present study did contain two-item scales assessing the importance to the individual of each subdomain. The response scale ranged from 1-4. Study participants reported a mean body importance rating of 3.30 . When discrepancy values were calculated after the method of Harter (1985) (i.e., body rating - importance rating), a mean body discrepancy rating of -.80 was obtained with a standard deviation of .88. Further analyses revealed that many of the most active women rated their bodies between 1 and 2 ( $R$. Sonstroem, personal communication, April 12, 1993). Therefore, it would seem that suppression effects in the present study involved suppressing the portion of Body variance that was unrelated to the dependent variables. This revealed a more substantive relationship between Body and physical activity in middle-aged women.

A refined model for predicting physical activity in adult women was tested by deleting Sport and Stren from the model, as well as their nonsignificant paths to dependent variables. The nonsignificant path from Eff was retained for theoretical purposes. The lower half of Table 3 contains goodness-of-fit indices, standardized regression coefficients, and percentages of dependent variable variance that were accounted for in these tests. The refined model was able to increase the CFI value from .911 to .927 . More importantly, the similarity in size and sign of the Cond and Body coefficients across analyses of the two models indicates that a majority of suppressor effects in the model's prediction of exercise are attributable to interaction between Body and Cond.

The data in Table 3 indicate that exercise in adult women is associated with high self-perceptions of physical condition and dissatisfaction with one's own body.

\section{Discussion}

The present research serves to enlarge the competence dimension of the EXSEM by partitioning the perceived physical competence level into a general 
physical self-worth level and a more specific, multidimensional subdomain level. This represents the second study demonstrating that people can discriminate across different levels of self-perception items classified by degree of generality. The study provides essential validity to the PSPP scales and to an expanded EXSEM hierarchy by use of confirmatory factor analysis and structural equation modeling.

In terms of intramodel associations, this study documented the necessity of including PSW within an expanded EXSEM. PSW served as a mediator between subdomains and SE. When its association with SE was nullified (Figure 1 , Model C), three of the subdomains failed to establish significant associations with SE. The one exception was Body. Model B failed to significantly improve the data fit provided by Model A. In terms of within-model relationships, restricting associations to adjacent levels (staircase effect) is parsimonious, and provides the greatest essential validity for the model in that it requires the presence of each model level.

EXSEM proved capable, also, of explaining up to $27.6 \%$ of the variance in self-reports of exercise. The pattern of component interaction with these variables outside the model was unexpected, however. As proposed by EXSEM theory, Eff should have developed the greatest correspondence with exercise participation. Several reasons are advanced for the fact that it did not. Although the latent variable, Eff, was developed for structural modeling purposes, the essential validity of a general self-efficacy construct has been questioned (Bandura, 1986; Shelton, 1990). Much of self-efficacy's early appeal and validity centered on the congruence of very specific self-perceptions and behavior.

Alternatively, Marsh, Walker, and Debus (1991) have questioned the ability of self-efficacy assessment to predict behavior as well as self-concept assessment. He believes that the strength and persistence of individual behavior is guided by internal frames of reference, evaluation, and affect, as well as by cognitive processes. Self-concept measurement seems more capable of activating these processes, whereas current self-efficacy assessment mainly emphasizes cognition. Finally, it must be recognized that the presence of suppressor effects diminished associations between Eff and exercise.

The presence of suppressor effects accentuated the close alliance between Body and other perceived physical competence components, most particularly Cond and PSW. Cond, with the largest zero-order relationship to exercise among the subdomains, developed an even larger multiple regression coefficient with exercise. It would seem that a portion of this enhanced coefficient would be attributable to the suppression present. The role of PSW in these interactions must be recognized also. PSW is directly associated with all subdomains in the model. PSW's overlap with Body has been a source of concern, and probably contributed to the suppression effect in this research. Body and PSW Pearson $r s$ from five samples in three studies have ranged from .71 to .80 (Fox \& Corbin, 1989; Sonstroem et al., 1992; this study). The present correlation corrected for attenuation was .85 (see Table 1).

It is suggested that this scale overlap may not represent a problem of instrument development but may be caused by a synonymity of terms in people's minds. Today's health-conscious society, besieged by lean, trim figures found in health and leisure advertisements, may equate an attractive body with health and physical self-worth. Additionally, many people appear to equate physical self-worth with physical attractiveness. 
Although this research should be replicated with subjects of different ages and backgrounds, it may not be premature to envision eventual modification of the PSPP and EXSEM. First, it may be profitable to investigate more intensively the form and manner in which physical self-worth varies across people. Ideally, it would seem possible to develop Body and PSW scales with less overlap. As discussed by Sonstroem and Morgan (1989), competence and self-acceptance represent the two major dimensions of self-esteem. Scales such as Body, PSW, and SE may be better considered as components of a self-acceptance or selflove dimension rather than as a mastery dimension. The Rosenberg Self-Esteem Scale is believed to assess a self-esteem dimension of self-acceptance (Crandall, 1973). It is quite possible that at higher levels of abstraction, self-love or selfacceptance represents the salient dimension rather than competence. Moreover, Bandura (1991) has seriously questioned Harter's employment of attractive body as a competence component. It is provocative that in this study's correlation matrix (see Table 1), SE, PSW, and Body develop the largest coefficients among themselves. Consideration of these elements as other than components of a mastery dimension entails assessment and model modifications.

Meanwhile, the PSPP and EXSEM remain very viable vehicles for increasing understanding in the area of exercise and self-perceptions. This research documents the reliability and validity of the PSPP for use with adult females, and it validates internal structure of EXSEM. Although we might expect different model elements to be linked with behavior in other populations (e.g., football or softball players), this study indicates that adult female aerobic dance and exercise activities are associated with positive self-evaluations of one's physical condition and with negative thoughts about one's body attractiveness.

\section{References}

Bandura, A. (1986). Social foundations of thought and action: A social cognitive theory. Englewood Cliffs, NJ: Prentice Hall.

Bandura, A. (1991). Conclusion: Reflections on nonability determinants of competence. In R.J. Sternberg \& J. Kolligian, Jr. (Eds.), Competence considered (pp. 315-362). New Haven, CT: Yale University Press.

Bandura, A., \& Adams, N.E. (1977). Analysis of self-efficacy theory of behavioral change. Cognitive Therapy and Research, 1, 287-308.

Bentler, P.M. (1989). EQS: Structural equations program manual. Los Angeles: BMDP Statistical Software.

Bentler, P.M. (1990). Comparative fit indices in structural models. Psychological Bulletin, 107, 238-246.

Cash, T.F., \& Green, G.K. (1986). Body weight and body image among college women: Perception, cognitive, and affect. Journal of Personality Assessment, 50, 290-301.

Cohen, J., \& Cohen, P. (1975). Applied multiple regression/correlation analysis for the behavioral sciences. Hillsdale, NJ: Erlbaum.

Crandall, R. (1973). The measurement of self-esteem and related constructs. In J. Robinson \& P. Shaver (Eds.), Measures of social psychological attitudes. Ann Arbor, MI: Institute for Social Research.

Fox, K.H. (1990). The Physical Self-Perception Profile manual. DeKalb, IL: Northern Illinois University, Office for Health Promotion. 
Fox, K.H., \& Corbin, C.B. (1980). The Physical Self-Perception Profile: Development and preliminary validation. Journal of Sport \& Exercise Psychology, 11, 408-430.

Harlow, L.L. (1985). Behavior of some elliptical theory estimators with nonnormal data in a covariance structures framework. Unpublished doctoral dissertation, University of California, Los Angeles.

Harter, S. (1985). Competence as a dimension of self-evaluation: Toward a comprehensive model of self-worth. In R.H. Leahy (Ed.), The development of the self. New York: Academic Press.

Huba, G.J., \& Harlow, L.L. (1987). Robust structural equation models: Implications for developmental psychology. Child Development, 58, 147-166.

James, L.R., Mulaik, S.A., \& Brett, J.M. (1982). Causal analysis: Assumption, models, and data. Beverly Hills, CA: Sage.

Marsh, H.W. (1990). Causal ordering of academic self-concept and academic achievement: A multiwave, longitudinal panel analysis. Journal of Educational Psychology, 82, 646-656.

Marsh, H.W., Walker, R., \& Debus, R. (1991). Subject-specific components of academic self-concept and self-efficacy. Contemporary Educational Psychology, 16, 331345.

Marsh, H.W., Byrne, B.M., \& Shavelson, R.J. (1988). A multifaceted academic selfconcept: Its hierarchical structure and its relations to academic achievement. Journal of Educational Psychology, 80, 366-380.

Messer, B., \& Harter, S.V. (1986). Manual for the Adult Self-Perception Profile. Denver: University of Denver Press.

Shavelson, R.J., Hubner, J.J., \& Stanton, G.C. (1976). Self-concept: Validation of construct interpretations. Review of Educational Research, 46, 407-441.

Shelton, S.H. (1990). Developing the construct of general self-efficacy. Psychological Reports, 66, 987-994.

Sonstroem, R.J. (1978). Physical Estimation and Attraction Scales: Rationale and research. Medicine and Science in Sports, 10, 97-102.

Sonstroem, R.J., Harlow, L.L., Gemma, L.M., \& Osborne, S. (1991), Test of structural relationships within a proposed exercise and self-esteem model. Journal of Personality Assessment, 56, 348-364.

Sonstroem, R.J., Harlow, L.L., \& Salisbury, K.A. (in press). Self-perceptions across a competitive swim season. Research Quarterly for Exercise and Sport.

Sonstroem, R.J., \& Morgan, W.P. (1989). Exercise and self-esteem: Rationale and model. Medicine and Science in Sports and Exercise, 21, 329-337.

Sonstroem, R.J., Speliotis, E.D., \& Fava, J.L. (1992). Perceived physical competence in adults: An examination of the Physical Self-Perception Profile. Journal of Sport \& Exercise Psychology, 14, 207-221.

Tabachnick, B.G., \& Fidell, L.S. (1983), Using multivariate statistics. Philadelphia: Harper and Row.

\section{Acknowledgment}

We wish to thank Wayne F. Velicer for his assistance on suppression issues.

Manuscript submitted: May 6, 1993

Revision received: September 12, 1993 